\section{Vitamin D Adequacy and Improvements of Comorbidities in Persons with Intellectual Developmental Disabilities}

\author{
Sunil J. Wimalawansa \\ Cardio Metabolic Institute, New Jersey, USA
}

Corresponding author:

Sunil J. Wimalawansa

झ suniljw@hotmail.com

The prevalence of vitamin D deficiency is alarmingly high among people with developmental disabilities. In addition to enhancing mineral metabolism, vitamin D affects neuro-modulation, muscle coordination, immunity, infections and autoimmune disorders. Peer-reviewed papers from PubMed, Medline, and other research databases were searched using key words to collate relevant evidence in preparation of this evidence-based review. Per the guidelines from the Preferred Reporting Items for Systematic Reviews and Meta-Analyses (PRISMA), general interpretations of results are included taking into consideration the risk-benefits, broader evidence, and implications for practicing clinicians. Many clinicians consider the minimum level of serum 25-hydroxy vitamin $D[25(\mathrm{OH}) \mathrm{D}]$ required for optimal health in the normal population to be $30 \mathrm{ng} / \mathrm{mL}$ ( $75 \mathrm{nmol} / \mathrm{L})$, whereas a lesser number consider the level to be $20 \mathrm{ng} / \mathrm{mL}$. However, evolving data suggest that for vulnerable populations, such as those with autoimmune disease, cancer, cardiovascular diseases, metabolic syndrome, and persons with developmental and intellectual disabilities, $40 \mathrm{ng} / \mathrm{mL}(100 \mathrm{nmol} / \mathrm{L})$ might be considered the minimum level. To reach this level, most people requires adequate sun exposure and/or a daily oral supplementation of between 1,000 and 5,000 International Units of vitamin $D$. This review explores vitamin $D$ requirements for the musculoskeletal and non-skeletal systems and its adequacy in relationship to common disorders affecting persons with intellectual disabilities.

Keywords: Autism; Autoimmune disorders; Diseases; Fractures; Falls; Institutionalized; Nursing homes; Residential; 25(OH) Vitamin D; Autism; Neurodevelopmental
Cardio Metabolic Institute, North Brusnwick, New Jersey, USA.

Tel: 7323056537

Citation: Wimalawansa SJ. Vitamin D Adequacy and Improvements of Comorbidities in Persons with Intellectual Developmental Disabilities. J Child Dev Disord. 2016, 2:3.

Received: April 28, 2016; Accepted: June 16, 2016; Published: June 23, 2016

\section{Introduction}

Adequate vitamin $D$ is essential for optimal health [1, 2]. Vitamin $D$ deficiency is one of the most common nutritional deficiencies in the world that can be easily treated. Emerging data suggest that the beneficial effects of vitamin $D$ extend beyond the regulation of calcium and phosphorus homeostasis. Although no randomized controlled clinical trial data exist, the increasing volume of data reported suggest the physiological importance of vitamin $D$ in systems other than the musculoskeletal system.

The prevalence of vitamin $D$ insufficiency is higher in certain vulnerable groups [3], such as those who are obese, those with impaired gastrointestinal absorption of vitamin $D$, those taking medications that enhance cytochrome P450 activity in the liver $[4,5]$, and persons who are institutionalized, such as in nursing homes $[6,7]$. People with disabilities, including longterm residents innursing homes, rehabilitation centers, Group Homes, and disability centres, have high prevalence of vitamin D deficiency [8-10]. Of the 30 leading causes of death (in 2010) in the United States, 19 have been linked to low vitamin D status [11]. These include cardiovascular disease, cancer, Alzheimer disease, diabetes mellitus, and falls and fractures in the elderly.

Because of multiple pathologies, coexisting disorders, and the intake of multiple medications that are known to enhance catabolism of vitamin D, persons with medically complex developmental disability (MCDD) [12] experience more comorbidities that are exacerbated by vitamin $D$ inadequacy. These patients are likely to require longer exposure to ultraviolet 
B-light (UV-B) and/or higher doses of vitamin D supplements to maintain healthy serum 25 -hydroxy vitamin $D[25(\mathrm{OH}) \mathrm{D}]$ levels.

Accumulating data also support the need to maintain a higher serum 25(OH)D level in persons with MCDD, than in healthy people for better health outcomes and to minimize comorbidities associated with vitamin D deficiency $[6,13]$.

The incidence and prevalence of vitamin $D$ deficiency and associated complications in persons with MCDD are different from those of the healthy population [14]. This review addresses the lack of knowledge regarding the relationships between serum vitamin D levels and musculoskeletal disorders and non-skeletal disorders, the potential for mistaken treatment of patients with osteomalacia with potent antiresorptive agents, and the relationship of vitamin $\mathrm{D}$ statuses with broader potential health outcomesin patients with MCDD.

\section{Common causes leading to vitamin D deficiency}

The persons with MCDD consists of groups of people with a variety of neurodevelopmental disorders and developmentally induced physical and intellectual disabilities. Limited data suggest a high incidence of vitamin $D$ deficiency among persons with MCDD $[15,16]$. Whether vitamin D deficiency aggravates existing comorbidities and/or initiates new conditions in this highly ulnerable population is not clear. Many patients with MCDD take multiple medications, such as anticonvulsant drugs and glucocorticoids, that enhancethe catabolism of vitamin D. Doing sofurther reduces the blood levels of vitamin $D$ and its stores [17]. This requires persons with MCDD to have a longer exposure to UV-B and/or higher doses of vitamin D supplements than the doses usually recommended for the general population to maintain serum vitamin D levels.

In the above group of persons, vitamin D inadequacy is attributable to a combination of insufficient sun exposure, suboptimal dietary intake and supplements, enhanced catabolism of vitamin $D$, and having multiple comorbidities. In general, less sun-exposure is in part attributable to staying predominantly indoors, concerns about skin cancer, climatic changes and related issues, pollution [18], lifestyle, obesity, and changes in dietetic patterns [19]. Figure 1 illustrates the natural cycle of generation of vitamin D. The figure also illustrates the importance of sun exposure as a natural means of generating endogenous vitamin $D$.

\section{Vitamin D requirements}

Many clinical studies of supplemental vitamin D have been nonconclusive because the provision of a substance to those who are already sufficient does not alleviate anything. To observe differences, intervention should be offered to those who are vitamin $D$ deficient [20]. Researchers have reported a wide range of healthbenefitsof vitamin $D[21,22]$. Nevertheless, only a small number of deficient and insufficient persons are supplemented with vitamin $D$. This is in part hampered by the high cost of measuring serum $25(\mathrm{OH}) \mathrm{D}$ and not making the right diagnosis [23].

When the rates of insufficiency and deficiency reach more than $70 \%$ in a community, one could argue (for cost-effectiveness purposes), that supplements can be offered (and to encourage sun exposure) in the absence (or in lieu) of measuring serum
$25(\mathrm{OH}) \mathrm{D}$ levels. Safe sun-exposure is the best option to raising serum vitamin D levels, but in those with MCDD, this often is not easy or practical.

Considering the logistical difficulties, costs, and the established higher safety limits of intake, every resident in high-risk community such as in a residential facility could be offered a combination of improved (supervised) safe sun-exposure,food fortification with vitamin $D$, and acceptable supplemental dose of vitamin D and other micronutrients. Considering the potential of significant reduction of falls and injuries including fractures, this approach is likely to be cost-effective.

\section{Meta-analyses data on the adverse effects of vitamin D}

In a meta-analysis of data that used vitamin $D$ doses from 400 7,000 IU per day or 8,400 - 54,000 IU per week with followup periods as long as 4 years, no adverse effects of vitamin D treatment were reported [24]. However, these studies were not designed to assess the true dose-responses or dose-related harm. None of these studies reported any differences of rates of serious adverse events, including hypercalcemia and hypercalciuria, or withdrawal because of adverse events [23, 24].

In another meta-analysis, none of the 17 trials included had a higher incidence of hypercalcemia in treated versus control participants $[4,25]$. The numbers of cases of hypercalcemia were low in both groups, and seven of the trials reported no cases of hypercalcemia or kidney stones, in vitamin-D-supplemented groups. Overall, studies suggest the safety of using vitamin D doses as high as $10,000 \mathrm{IU}$, daily $[4,25]$.

The upper daily safe limit for average healthy people is considered 5,000 IU a day, and toxicity does not develop until serum levels reach more than $125 \mathrm{ng} / \mathrm{mL}$ (>312 nml/L) [4, 25]. Toxicity may manifest in persons who consume vitamin $D$ in doses excess of 50,000 IU daily for several months. Toxicity would not appear unless serum 25(OH)D levels exceeds and sustained above 100 $\mathrm{ng} / \mathrm{mL}(250 \mathrm{nmol} / \mathrm{L})$ for a period [26].

Thus, it is safe to treat individual residents in developmental disability facilities, using orally administered doses of vitamin $D$ between 2,000 and 4,000 IU a day or 50,000 IU twice a month; adverse effects are caused by much higher doses [8, 25, 27].

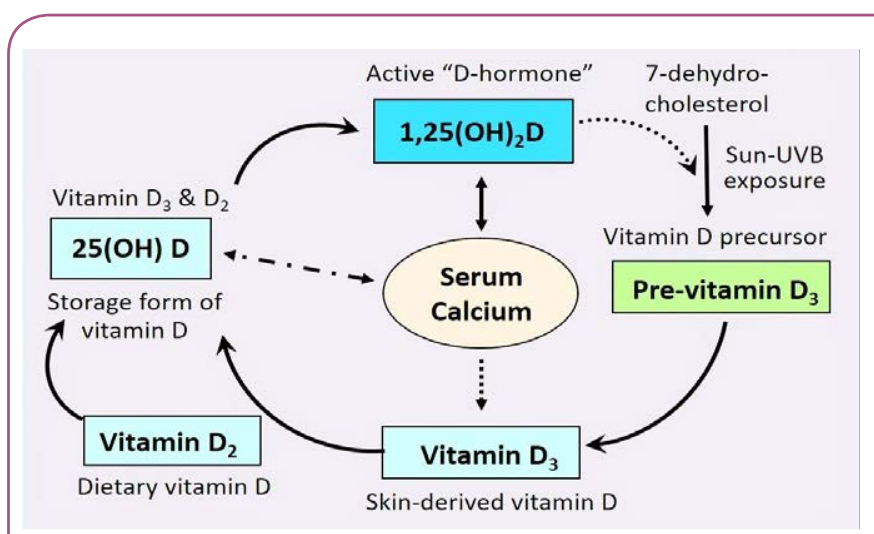

Figure 1

The generation of pre-vitamin $\mathrm{D}, 25(\mathrm{OH}) \mathrm{D}$, the activation steps of $25(\mathrm{OH}) \mathrm{D}$ to the active hormonal form, 1,25(OH)2D, and their inactivation steps are illustrated [27]. 


\section{Guidelines and normal ranges}

The rate of diagnoses for vitamin D deficiency has markedly increased since 2007 [28]. Most diagnoses concluded were for non-specific diseases suggesting that measurements were ordered as a screening process, instead as a diagnostic process.

In recent years, several organizations [e.g., The Institute of Medicine (IOM) [29], The Endocrine Society (TES) [4], The American Geriatrics Society [30], American Academy of Developmental Medicine and Dentistry [14], and ad hoc vitamin $D$ groups $[22,31]$ have developed clinical practice guidelines.

However, based on the articles reviewed and included in the guideline document and the intended population(s), the recommendations vary. For example, the IOM guideline defined vitamin D insufficiency as serum 25(OH)D levels between 11 and $20 \mathrm{ng} / \mathrm{mL}$ and vitamin D deficiency as a $25(\mathrm{OH}) \mathrm{D}$ below $10 \mathrm{ng} /$ $\mathrm{mL}$ [29]. TES defined vitamin D deficiency as a $25(\mathrm{OH}) \mathrm{D}$ level less than $30 \mathrm{ng} / \mathrm{mL}$ and vitamin $D$ insufficiency as a level between 21and $29 \mathrm{ng} / \mathrm{mL}$ [4].

The IOM recommendations were intended for a healthy population in North America for making public health decisions [29] and not intended to be applied in the treatment of patients [32]. The TES guidelines are for managing individuals and patients in general [4]. However, neither set of recommendations addressed vulnerable groups or subgroups of patients. The latter groups require special guidelines and in most cases likely to require a higher minimum serum $25(\mathrm{OH}) \mathrm{D}$ level than that recommended levels by TES, $30 \mathrm{ng} / \mathrm{mL}[4,14,19,25,33]$.

Many experts agree that the minimum serum 25(OH)D level of $30 \mathrm{ng} / \mathrm{mL}$ is adequate for normal healthy individuals, but highrisk populations, including persons with MCDD, likely need the optimal serum vitamin D levels greater than $40 \mathrm{ng} / \mathrm{mL}[14,17$, 27], as discussed later in this text. In this regard, in two separate African tribes examined, the pastoral Masai and the huntergatherer Hadza tribes, the average serum $25(\mathrm{OH})$ vitamin D level was $46 \mathrm{ng} / \mathrm{mL}$, suggesting that optimal serum $25(\mathrm{OH}) \mathrm{D}$ level is beyond $40 \mathrm{ng} / \mathrm{mL}[34,35]$.

It is important to understand that those with MCDD are at high risk for multiple chronic health issues, some of which may be alleviated in the presence of vitamin $D$ adequacy. Considering that theoverwhelmingmajorityof MCDD persons are vitamin $D$ deficient, The American Academy of Developmental Medicine \& Dentistry (AADMD) commissioned a Vitamin D-Task Force to develop specific guidelines and recommendations for persons with intellectual and developmental disability.

The Task Force recommended minimum serum 25(OH) D levels of $40 \mathrm{ng} / \mathrm{mL}$ for persons with MCDD [14]. The Task Force recommended that 25(OH)D concentrations needed for optimal health in persons with MCDD are between 30 and $50 \mathrm{ng} /$ $\mathrm{mL}(75-125 \mathrm{nmol} / \mathrm{L})$, which can be achieved safely using orally administered vitamin D3 supplements up to 4,000 IU/day and/or sensible exposure to solar UVB radiation [14].

\section{Vitamin D supplementation}

Many residents in developmental disability centers receive between 400 and $600 \mathrm{IU}$ of vitamin D a day through their pharmacies. Since the diet provides too little vitamin $D$, in the absence ofsun exposure, such small doses enable very few residentsto achieveserum 25(OH)D levels beyond $30 \mathrm{ng} / \mathrm{mL}$. In addition, most persons with developmental and neuro-disabilities receive a number of medications that enhance the catabolism of vitamin $D[8,27,36]$.

Several publications support the necessity of having serum $25(\mathrm{OH})$ $D$ levels greater than $40 \mathrm{ng} / \mathrm{mL}$ to alleviate certain diseases. Such diseases include some diseases affecting the musculoskeletal system [25], management of breast [37] and other cancers [38], Crohn's disease [39], type 1 diabetes [40], type 2diabetes [41], infections [42], multiple sclerosis [43], and systemic lupus erythematosus [44].

Persons with MCDD who have multiple comorbidities are also likely to require higher intake of vitamin $\mathrm{D}$ (e.g., between 2,000 and 5,000 IU daily to keep the serum $25(\mathrm{OH}) \mathrm{D}$ levels between 40 and $60 \mathrm{ng} / \mathrm{mL}$ ) than does the general population to maintain serum vitamin D levels above $30 \mathrm{ng} / \mathrm{mL}[14,32]$.

Most people with MCDD living in disability centers are vitamin $D$ deficient, but a few have adequate vitamin D levels. However, giving vitamin D doses such as 4,000 IU a day or 50,000 IU twice a month is highly unlikely to raise the serum vitamin $D$ levels above $60 \mathrm{ng} / \mathrm{mL}$ even in those with adequate levels before supplementation. Levels greater than $125 \mathrm{ng} / \mathrm{mL}$ are considered toxic $[4,31]$. In addition to the broader benefits from vitamin $D$ adequacy and the disadvantage of high cost of vitamin $D$ testing, many patients with MCDD would not cooperate with venipuncture. These factors support the argument that persons with MCDD could be safely treated with routine oral vitamin D3 supplementation.

\section{Cost-effective approaches to alleviate vitamin D deficiency in institutionalized persons}

For most people in the world, diets do not provide more than 500 IU of vitamin per day, but the daily requirements are much higher $[45,46]$. In the absence of adequate exposure to UVB, many people would not be able to obtain adequate vitamin $D$ for optimal physiological functions. For example, using the TESrecommended minimum level of vitamin $D$ of $30 \mathrm{ng} / \mathrm{mL}$, more than $50 \%$ of children and adults in North America were reported as vitamin $D$ insufficient $[27,47,48]$, in part, because of sun avoidance and inadequate UVB availability during the winter months. Thus, in the absence of adequate exposure to UVB rays, many become vitamin $D$ insufficient or deficient. The prevalence is particularly high among persons with MCDD and those who are housebound.

For most persons with MCDD, the administration of vitamin D at doses less than 1,000 IU a day or 50,000 IU a month, is insufficient to achieve serum $25(\mathrm{OH}) \mathrm{D}$ levels greater than $30 \mathrm{ng} /$ $\mathrm{mL}$ (75 nmol/L) [49]. Because of the marked fluctuation of serum levelswhen vitamin Dis administered at infrequent than monthly intervals, such a regimen is less beneficial even though it may help to attain the target levels of serum $25(\mathrm{OH}) \mathrm{D}$ for a short period $[25,33,50]$. However, the goal should be to maintain the desirable, steady-state serum $25(\mathrm{OH}) \mathrm{D}$ level, throughout the year. 
Routine testing of serum vitamin $D$ is inconvenient for persons with MCDD. It only increases cost withlittlebenefit. For this particular population, administering one capsule of 50,000 IU vitamin D3 once every 2 weeks ( $\$ 0.30$ per capsule $\times 26$ capsules per year) would cost less than $\$ 10$ per year.

Considering virtuallyno adverse effects with such a dose and the potential benefit in decreasing morbidities, this is a highly costeffective and practical approach in this population.

\section{Key actions of vitamin D on musculoskeletal health}

Vitamin D facilitates intestinal absorption of calcium and maintains optimal skeletal health. It is also necessary for mineralization and maintenance of the skeleton, proper reflexes, and neuromuscular coordination. Even subclinical vitamin D deficiency can increase sway and falls in the elderly and vulnerable people [51]. In addition, it may exacerbate osteoporosis through vitamin D-deficiency-induced secondary hyperparathyroidism and consequent increased osteoclastic bone resorption [19, 52, 53], thus increasing fracture risks.

In addition, an inverse relationship exists between serum PTH and 25(OH)D levels up to about $50 \mathrm{ng} / \mathrm{mL}$, plateauing beyond that $[54,55]$. Higher PTH levels are associated with increased morbidity and mortality, in part because of increased incidences of cardiovascular disease [56-61]. Figure 2 illustrates an inverse relationship of serum 25(OH)D and serum PTH levels. Vitamin $D$ is important for all tissues in the body and the development and mineralization of bones. Rickets and osteomalacia are manifestations of severe, chronic vitamin D deficiency. In children, vitamin D deficiency causes rickets, resulting in softening of bones, leading to skeletal deformities. In adults, it leads to osteomalacia, muscular weakness, falls, and fractures [62-69]. Compared with the healthy population, people with MCDD have more comorbidities, including osteomalacia [9, 70] and osteoporosis [71-73], and increased incidence of falls and fractures [74-76].

\section{Use of antiresorptive agents in persons with MCDD}

In recent years, the potent antiresorptive agents, bisphosphonates and denosumab have become popular for the treatment of low bone mineral density (BMD) as measured by dual-energy $x$-ray absorptiometry (DXA). This is particularly disturbing in institutionalized adults with intellectual disabilities, who have a high incidence of osteomalacia. Considering that patients with vitamin $D$ deficiency often experience poor response to bisphosphonates, it is questionable whether many of these patients need or benefit from (or may be even harmed by) treatments with potent anti-osteoporosis pharmaceuticals [72].

For example, in an observational study, favorable response to bisphosphonates was seen more frequently in patients with serum $25(\mathrm{OH}) \mathrm{D}$ levels greater than $33 \mathrm{ng} / \mathrm{mL}$ [77]. In this study, those with 25(OH)D levels less than $30 \mathrm{ng} / \mathrm{mL}$, only $21 \%$ responded, $50 \%$ responded in those with levels between 30 and $40 \mathrm{ng} / \mathrm{mL}$, and $77 \%$ responded in those with serum $25(\mathrm{OH}) \mathrm{D}$ levels greater than $40 \mathrm{ng} / \mathrm{mL}$, as documented by increasing BMD [77]. Data suggests an increased BMD associated with increasing levels of serum vitamin $D$, following anti-osteoporosis therapy;

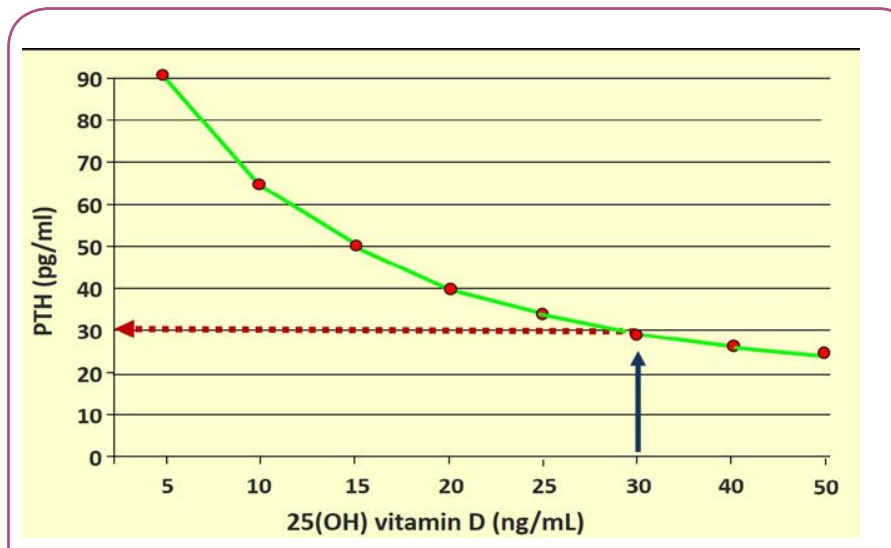

Figure 2 Inverse relationship between the serum 25(OH)D and serum intact PTH (parathyroid hormone) levels. Median values are given, and individual scatter data points have been removed to improve clarity (median data from a number of studies) [183-185] [graph was modified from [27]]. With increasing serum $25(\mathrm{OH}) \mathrm{D}$, the serum PTH levels continue to decrease below $30 \mathrm{pg} / \mathrm{mL}$ and seem to stabilize around $20 \mathrm{nmol} / \mathrm{L}[46,54,55]$.

however, this needs to be confirmed by other studies.

\section{Misinterpretation of BMD data leading to mistreatment in patients with MCDD}

Age has a profound impact on bone loss and the risk of fractures. As a result, the prevalence of osteopenia and osteoporosis as demonstrated by measuring BMD increases with advancing age in all skeletal sites that are vulnerable to fragility fractures [53]. Because most persons with MCDD are relatively inactive, their peak-bone mass and the BMD is lower, and have a higher risk of fractures. However, just because the BMD is low does not mean that a person is osteoporotic, particularly in the MCDD population, in whom the prevalence of vitamin D deficiency and osteomalacia are high. Consequently, instead of having their vitamin D deficiency and underlying osteomalacia corrected, many of these patients are treated inappropriately with potent antiresorptive agents, such as bisphosphonates and denosumab, because vitamin $D$ deficiency is not diagnosed. This preventable error should be avoided with future medications, such a odanacatib. In this population, many have low BMD in coexistence with osteomalacia and thus, should be treated with vitamin $D$

supplements first to correct their vitamin D deficiency. BMD measurements obtained using DXA scanning cannot differentiate osteoporosis form osteomalacia. If the lower BMD is primarily caused by osteomalacia, correction of vitamin D deficiency would rapidly increase BMD by facilitating mineralization of existing uncalcified osteoid. Therefore, in these patients the "diagnoses" of osteopenia and osteoporosis are being made erroneously, using numerical BMD values generated though DXA, which is not capable of differentiating osteomalacia from osteoporosis. Therefore, in the absence of correcting vitamin D deficiency and associated osteomalacia, the uses of potent antiresorptive agents can be harmful. Neither the DXA machine nor physicians who 
interpret these data can differentiate between osteomalacia and osteoporosis based on BMD data (i.e., grams of mineral per square centimeter or the T-scores) [27]. As a result, when these patients are mistakenly labelled as having osteoporosis, physicians are inclined to treat them with potent antiresorptive agents, such as bisphosphonates, denosumab (or odanacatibindue course), which is unscientific and inappropriate.

\section{Importance of correcting vitamin D deficiency before starting antiresorptive therapy}

Underlying vitamin D deficiency should be corrected by giving adequate doses of vitamin $D$, before considering the use of antiresorptive therapy [8]. After improving the serum vitamin D levels to an optimal range (e.g., greater than $40 \mathrm{ng} / \mathrm{mL}$ in persons with MCDD), it is reasonable to repeat the DXA scan in 6 months or a year later, using the same machine. If the BMD remains low after correction of vitamin $D$ deficiency and there are no additional correctable secondary causes present, it is reasonable to consider prescribing an anti-osteoporosis agent, if the patient is at high risk for fractures.

Unfortunately, because of the lack of a basic understanding of underlying pathophysiology, a large number of patients with MCDD are treated with potent anti-osteoporosis therapies that may not benefit but harm them. In the presence of vitamin $D$ deficiency, such therapies have little or no benefit on fracture reduction. In fact, such therapy may aggravate or precipitate osteomalacia, develop hypocalcemia, and worsen seizures. In addition, the underlying issue of vitamin $D$ deficiency continues to increase comorbidities.

\section{Persons with MCDD have higher incidence of falls and fractures}

Postural swing has been linked to increased risk of falls in those with serum 25(OH)D levels below $12 \mathrm{ng} / \mathrm{mL}$. Treatment with vitamin $D$ improved sway and decreased the incidence of falls in the elderly [78]. People with MCDD often have osteomalacia [9], osteopenia, or osteoporosis [70] and low bone mass [33,79]; collectively, all of these increase fracture risks. Having strong bones and good neuromuscular control are the key to minimizing falls and fractures.

For example, in individuals with vitamin $D$ deficiency and increased postural sway, treatment with 8,400 IU of vitamin D3 once a week reduced the sway [80]. Evidence from other randomized clinical trials supports that vitamin D supplements improve muscle strength and overall function of the elderly and reduce the risk of falls in the elderly. These findings support the notion that vitamin D supplements improve overall neuromuscular function and coordination [81].

Falls among disabled people occur frequently, especially in those with low serum 25(OH)D levels [82]; such people sustain a higher incidence of fractures [83]. The combination of disabilities and vitamin $D$ deficiency further increases the risk of falls $[6,13,84]$.
This is a major issue in persons with neurodisabilities, who are more vulnerable to fall and fracture $[36,73,75,85,86]$.

\section{Falls can be reduced with the correction of vitamin D deficiency}

The frequency of falls in the elderly and disabled can be reduced by correcting vitamin D deficiency [80]. Comorbidities, including the consumption of certain medications (e.g., psychotropic drugs), visual and hearing impairment, axial orthopedic disabilities, peripheral nerve dysfunction, balance issues, and muscle weakness, increase fall risks [87]. In the MCDD population, muscle weakness and poor coordination are particularly important risk factors for falls and fractures that decrease with vitamin Dsupplementation [78, 87-100]. Thus, it is important to address this issue in all osteoporosis and fracture-prevention programs, and by geriatricians $[8,19,53]$.

A meta-analysis of double-blind, randomized clinical trials revealed that supplementation with vitamin $D$ reduced the fall risks in institutionalized individuals by more than $20 \%$ [87-89, $101,102]$. Thus, fall reduction must be given a priority in all residential care facilities. Several meta-analyses of randomized clinical trials confirmed a significant reduction of falls risk with vitamin D supplementation [98, 103, 104]. Therefore, optimizing serum 25(OH)D levels above the minimal recommended levels using vitamin D supplementation or by UVB exposure in patients with MCDD should be considered as standard of care in clinical practice.

In addition to vitamin D supplements, corrective measures should include environmental modifications, including removal of hazards and obstacles, the provision of appropriate railings, hearing aids, and corrective eyeglasses. In addition to vitamin D deficiency, arthritis, visual and hearing impairment, central or peripheral neuralgic dysfunction, and peripheral neuropathy increase the risk of falling. Although some researchers have suggested that analogues of vitamin $D$ may be more effective than vitamin D for fall prevention [105], this has not been proven in other clinical studies.

\section{Correcting vitamin D deficiency}

Available doses of vitamin D3 range from 1,000-50,000 IU as gel capsules; they are inexpensive and well tolerated. Adequate doses improve muscle function and reflexes and thereby effectively prevent falls and fractures [78, 87-100].

Vitamin D plays an important role in neuromuscular control, which reduces the risk of falls and consequently fractures [106]. Improving vitamin D status is an important modifiable risk factor for reducing falls and fractures [80], particularly in those with MCDD. Because of the relatively shorter half-life of vitamin D2, vitamin D3 is preferred and should be administered no less frequently than once monthly $[8,19]$.

Vitamin D may come from UV exposure, dietary intake, or supplements. The input from all sources required each day to meet physiological needs and support optimal health is estimated to be roughly $6,000 \mathrm{IU} /$ day $[107,108]$. It has been estimated that raising the minimum year-round serum $25(\mathrm{OH}) \mathrm{D}$ level between 
40-60 ng/mL (100-150 nmol/L)would prevent approximately 58,000 new cases of breast cancer and 49,000 new cases of colorectal cancer each year [109]. Such levels are also expected to reduce the case-fatality rates by half, in patients with breast, colorectal, or prostate cancer [110]. There is no significant risk associated with taking 2,000-5,000 IU vitamin D3 per day or increasing the population serum $25(\mathrm{OH}) \mathrm{D}$ level to greater than $40 \mathrm{ng} / \mathrm{mL}$.

\section{Extra-skeletal benefits of vitamin D}

Hundreds of research reports indicate that 25(OH)D has a number of beneficial effects on the extra-skeletal tissues, including the and cardiovascular system [111-116]. Most of the relationships reported between serum 25(OH)D and extra-skeletal benefits are associations and do not provide "cause-effect" relationship [117]; however, supportive evidence continues to accumulate.

One needs to be cognitcient that vitamin $D$ deficiency could be a "marker" of poor nutrition and/or ill health [7, 8, 27, 84]. These associations can be affected by many confounding factors, such as place of living, diet, smoking, and supplements [29, 118]. In contrast to the aforementioned, data from two recent systematic reviews found no evidence that vitamin $D$ supplements modify extra-skeletal conditions $[119,120]$. Figure 3 illustrates the associations and interactions between vitamin $D$ and various disorders.

\section{Common disorders that are aggravated by vitamin deficiency}

Muscle weakness and sarcopenia have an inverse correlation with serum $25(\mathrm{OH}) \mathrm{D}$ levels [80]. There are abundant vitamin $\mathrm{D}$ receptors (VDR) in muscle cells that increase with physical activity [121]. However, the expression of muscle VDR levels declines with age [92] and is associated with sarcopenia. In addition to aging, inactivity and vitamin $D$ deficiency contribute to the development of muscle weakness and sarcopenia, enhancing fall risks; these can be reversed with vitamin $\mathrm{D}$.

Epidemiological studies have suggested links between serum 25(OH)D levels (and exposure to sunlight) and the incidences of breast [122-124], colon (digestive tract), prostate, and ovarian cancers; non-Hodgkin's lymphoma; and certain leukemias [111, 125-130]. However, whether the incidence of cancer would decrease with normalization of vitamin D status is not known.

Persons with MCDD are predisposed to a higher incidence of infections, perhaps as a consequence of impairment of the immune system secondary to vitamin D deficiency, which enhances the susceptibility to viral and bacterial infections, including tuberculosis $[131,132]$. Evidence supports that adequate serum vitamin $D$ levels enhance immunity. Indirect bactericidal effects of vitamin $D$ that manifest via the induction of cathelicidin may also contribute to this [133]. Research alsosuggests an association between low serum vitamin $D$ levels and inflammatory bowel diseases [134-137].

\section{Other muscular and neurological defects}

Persons with MCDD have a high incidence of swallowing difficulties and neurological defects. Dysphagia is common among persons with developmental disabilities for a number of reasons, including the widespread use of neuroleptic medication, abnormal posture, non-cooperation in swallowing, yeast infections, and gastroesophageal reflux disease. Dysphagia can lead to aspiration pneumonia, malnutrition, and sarcopenia. Although a connection between vitamin D deficiency and swallowing deficits is biologically plausible, there are no causative data available on vitamin D status and the risk of dysphagia.

Considering the high prevalence of vitamin D deficiency in persons with MCDD, it is plausible that decreased muscle function and coordination caused by vitamin $D$ deficiency may be causally related to the increased incidence of dysphagia observed [138, 139]. In addition, suboptimal vitamin D levels may affect the severity of over-active bladder in patients with chronic disabilities. Because of inactivity and muscle weakness, some people with MCDD develop abdominal obesitythat worsen in the presence of vitamin D deficiency[140].

Pulmonary function is known to worsen in those with vitamin D deficiency. Regarding chronic lung diseases, whether the decline of respiratory muscle function is attributable to vitamin D deficiency, chronic silent aspiration from oropharyngeal dysfunction, chronic obstructive airway disease and chronic inflammation, or impaired lung tissueremodeling, is not known $[141,142]$.

VDR and vitamin D-associated enzymes are expressed in the central nervous system, particularly in areas affected by neuro-degenerative disorders, especially in the hippocampal region[143]. Vitamin D deficiency has also been correlated with worsening Alzheimer disease and memory, both of which can lead to increased falls and fractures [144].

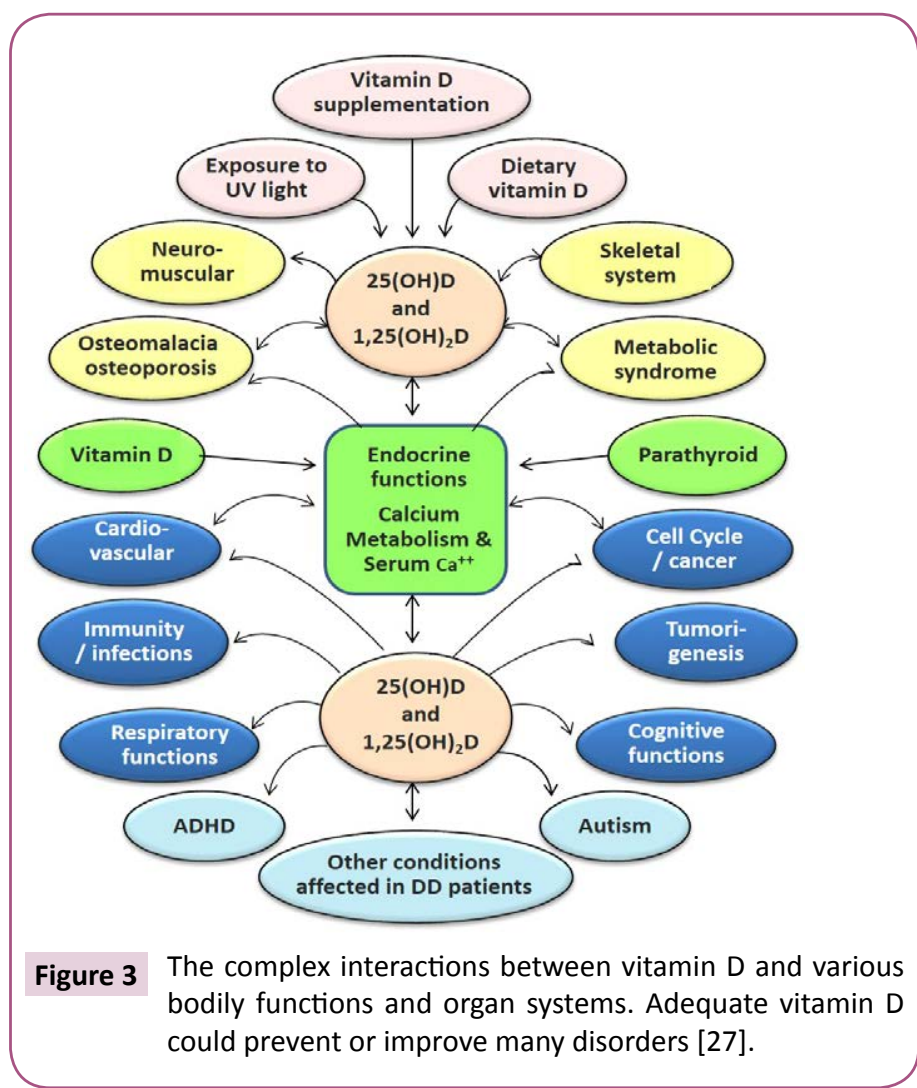




\section{Autism, attention deficit syndrome, and oral health}

Accumulating community-based evidence indicates that vitamin $D$ deficiency may enhance the risk for and the progression of autism $[85,145,146]$. A study of children in the United States reported significant inverse correlations of solar UVB exposure (a proxy for vitamin D production) with an increased prevalence of autism [145]. It has been suggested that vitamin D may regulate cerebral serotonin synthesis, which may lead to an increased risk of autism [147]. While this may not be the only mechanism, whether correcting vitamin D status would improve the symptoms of autism remains unknown [85].

People with intellectual and developmental disabilities have an increased incidence of attention deficit-hyperactivity disorders (ADHDs) [148]. Affected persons are known to have lower serum $25(\mathrm{OH}) \mathrm{D}$ levels than do control subjects [149, 150]. A larger, community-based randomized clinical trials are needed to assess whether vitamin $D$ supplementation would alleviate the symptoms of ADHDs [151]. There are many theories regarding the beneficial effects of vitamin $D$ in the alleviation of developmental disorders that affect the central nervous system; these need further investigation.

Poor oral hygiene and dental caries are common among persons with developmental disabilities. A recent meta-analysis of controlled clinical trials reported significant benefit of vitamin $D$ supplementation on oral health [152]. Vitamin D is also shown to reduce the risk of dental caries [153]. Nevertheless, low vitamin $D$ levels could be a surrogate marker for poor oral hygiene. Also be in the and low socioeconomic status, thus, unaffordability and lack of access (insurance issues) to dentists and oral hygienists. Vitamin D deficiency has also been shown to be associated with premature tooth loss and escalating periodontal diseases [154, 155].

\section{Other conditions commonly present in persons with MCDD}

In addition to common autoimmune disorders, multiple sclerosis [156], Gaucher disease (type 1-lysosomal storage disorder) [157], Williams syndrome [73], Down syndrome [72, 75], and many other disorders are known to be associated with low serum $25(\mathrm{OH}) \mathrm{D}$ levels $[36,72,75]$. There are ongoing studies to test the postulated associations between various diseases with vitamin $D$. Other studies have demonstrated an inverse relationship between $25(\mathrm{OH}) \mathrm{D}$ levels and the all-cause mortality rates $[158,159]$ and frailty $[160,161]$.

Meta-analysis data show that an increase of serum $25(\mathrm{OH})$ D levels from $22 \mathrm{ng} / \mathrm{mL}$ to $42 \mathrm{ng} / \mathrm{mL}$ (54 to $105 \mathrm{nmol} / \mathrm{L}$ ) was associated with a statistically significant, $15 \%$ reduction in incidence of cardiovascular diseases [109] and decrease of breast and colorectal cancer incidences by $30 \%$ (Table 1)[38, 162].

\section{Discussion}

It is important to note that not all vitamin D-related clinical studies are positive. Although some are ambiguous, few showed harm when individuals were treated with high doses of vitamin D, but such is a minority [176-178]. This review describes pros and cons of measurements and required blood levels of vitamin $D$ and provides recommendations for supplementation of vitamin $D$ in persons with intellectual disabilities.

Potential vitamin $D$ responsive conditions of relevance to those with neuro-developmental disorders and intellectual disabilities include bone metabolism, falls and fractures, physical functioning, infectious diseases (including tuberculosis and respiratory infections), insulin resistance, type 2 diabetes mellitus, cardiovascular diseases, Alzheimer disease, autism, ADHDs, cancer, oral health, and reduction of all-cause mortality.

Evidence suggests that the administration of vitamin $D$ supplements at intervals of greater than once a month has less beneficial effects (and perhaps harm) in preventing falls and fractures (Figure 3)[178-180]. Considering the relatively short half-life of vitamin $D$, unphysiological fluctuatons of serum and intra-cellular vitamin $D$ levels could explainless benefits with infrequent administration of oral or parenteral vitamin D.

To alleviate various diseases and disorders requires different serum 25(OH)D levels, with certain diseases, such as cancer and autoimmune disorders, requiring higher levels (Table 1). Nevertheless, as with other physiological phenomena, the goal should be to achieve the optimal necessary serum 25(OH)D levels to obtain the most benefit in body tissues to reduce vitamin D-deficiency associated disorders while minimizing potential, albeit rare, adverse effects [181]. Thus, aiming to achieve the middle of the suggested normal range (optimal level) $(50 \mathrm{ng} / \mathrm{mL}$ ) should provide the benefit of achieving serum $25(\mathrm{OH}) \mathrm{D}$ levels above $40 \mathrm{ng} / \mathrm{mL}$ in most persons with MCDD. Supplementation with vitamin $D$ should be considered together with other micronutrient supplements in vulnerable populations. It can be conveniently provided through food fortification programs [182] to cost-effectively overcome specific diseases or disorders [164].

Table 1 illustrates a simplified version for understanding the minimum serum levels of $25(\mathrm{OH}) \mathrm{D}$ required to overcome various ailments in broader subgroups of people.

\begin{tabular}{|c|c|c|}
\hline Disease or Disorder & $\begin{array}{l}\text { Minimum Serum } \\
\text { 25(OH)D Required }\end{array}$ & References \\
\hline Osteomalacia & $\begin{array}{c}\text { Serum } 25(\mathrm{OH}) \mathrm{D} \text { levels } \\
15 \mathrm{ng} / \mathrm{mL}\end{array}$ & $\begin{array}{c}{[22,31,163} \\
164]\end{array}$ \\
\hline $\begin{array}{l}\text { Muscular functions Fall } \\
\text { prevention Dental health and } \\
\text { oral hygiene Cardiovascular } \\
\text { protection All-cause mortality }\end{array}$ & $\begin{array}{l}\text { Serum } 25(\mathrm{OH}) \mathrm{D} \text { levels } \\
30 \mathrm{ng} / \mathrm{mL}\end{array}$ & $\begin{array}{c}{[22,31,164-} \\
170]\end{array}$ \\
\hline $\begin{array}{c}\text { Prevention of autoimmune } \\
\text { disorders, cancer, osteoporosis, } \\
\text { type } 2 \text { diabetes, infections and } \\
\text { obesity }\end{array}$ & $\begin{array}{l}\text { Serum } 25(\mathrm{OH}) \mathrm{D} \text { levels } \\
40 \mathrm{ng} / \mathrm{mL}\end{array}$ & $\begin{array}{c}{[22,31,162,} \\
164,166 \\
171-175]\end{array}$ \\
\hline $\begin{array}{l}\text { Subgroups of people who } \\
\text { would be benefited by } \\
\text { maintaining their serum } 25(\mathrm{OH}) \\
\text { D levels above } 40 \mathrm{ng} / \mathrm{mL}\end{array}$ & $\begin{array}{l}\text { Those who are obese, } \\
\text { have metabolic } \\
\text { syndrome or } \\
\text { autoimmune disorders, } \\
\text { those in residential- } \\
\text { care facilities, and } \\
\text { those with intellectual } \\
\text { and developmental } \\
\text { disabilities }\end{array}$ & $\begin{array}{c}{[22,31,70,} \\
162,164,166, \\
171-175]\end{array}$ \\
\hline
\end{tabular}


Because sick people spend more time indoors (thus, producing less vitamin D through exposure to UVB) and live mostly a sedentary lifestyle, the validity of the associations reported between vitamin $D$ and various diseases is not clear. Although lower vitamin $D$ levels are associated with a variety of illnesses, as demonstrated in observational studies, with the exception of musculoskeletal disorders (bone diseases and falls) [183], there currently is not enough evidence from randomized clinical trials that vitamin D supplementation prevents such illnesses.

Persons with MCDD will be benefited by maintaining their serum $25(\mathrm{OH}) \mathrm{D}$ levels above $40 \mathrm{ng} / \mathrm{ml}(100 \mathrm{nmol} / \mathrm{L})$ throughout the year [14], with the recommended range of 40 to $60 \mathrm{ng} / \mathrm{mL}$. Expected7 benefits include elimination of osteomalacia, improvement in mental and physical well-being, lower PTH levels, promotion of healthy BMD with normal bone architecture, reduction of the risks of falls and fractures, enhanced efficacy and safety of antiosteoporosis medications (if needed), reduction of the burden of several disorders that are associated with vitamin D deficiency, and relief from a host of other non-skeletal beneficial effects with minimal cost.

\section{Conclusions}

Routine measurement of serum 25(OH)D levels (and associated expenses) may not be justifiable in situations in which most people are likely to be vitamin $D$ deficient, as in the case of residents in nursing homes and developmental disability facilities [10]. Moreover, vitamin D intakes of $\leq 5,000 \mathrm{IU} /$ day do not cause demonstrable adverse effects in adults, while the safe upper limit is considered as $10,000 \mathrm{IU} /$ day.
Considering the broader benefits, cost-effectiveness and minimal adverse effects, we encouraged targeting serum 25(OH)D levels to $40 \mathrm{ng} / \mathrm{mL}$ (100 pmol/L) as the lower limit for persons with intellectual disabilities, with the recommended range of $40-60$ $\mathrm{ng} / \mathrm{mL}$. This can be achieved in over $98 \%$ of people with oral vitamin D3 supplements of less than 5,000/day (between 2,000 and 4,000 IU/day) or one gel-caps of 50,000 IU, administer orally, once in two weeks. Optimizing serum vitamin $D$ levels to above $40 \mathrm{ng} / \mathrm{mL}$ is likely to decrease burdens from comorbidities and improve overall health.

To avoid fluctuations of serum 25(OH)D levels, vitamin D3 should be administered at a frequency of less than once a month. When there is severe vitamin D deficiency, an appropriate oral loading of dose D3 should be considered [e.g., 4 to 6, 50,000 IU oral capsules or an injectable vitamin D vial containing $\sim 300,000 \mathrm{IU}$, preferably administered orally (instead of giving IM injections) which would avoid pain and reaching supra-pharmacological vitamin D levels in blood, is a low-cost effective option availableoutside USA]. Unless there is a severe deficiency or major issues with intestinal absorption, parenteral administration should be discouraged.

\section{Acknowledgments}

The author thanks Drs. Philip May and Matt Holder for their assistance in an earlier version of this manuscript.

\section{Conflicts of Interest}

This research received no grant from any funding agency in the public, commercial, or not-for-profit sectors. The author has no conflicts of interest. 


\section{References}

1 Vieth R (2006) What is the optimal vitamin D status for health? Prog Biophys Mol Biol 92: 26-32.

2 NIH (2010) Dietary supplement fact sheet: vitamin D. [http://ods. od.nih.gov/factsheets/vitamind.asp].

3 Choudhary A (2013) Prevalence of vitamin D insufficiency in survivors of childhood cancer. Pediatr Blood Cancer 60: 1237-1239.

4 Holick MF (2011) Evaluation, treatment, and prevention of vitamin D deficiency: an Endocrine Society clinical practice guideline. J Clin Endocrinol Metab 96: 1911-1930.

5 Holick MF (2012) Evidence-based D-bate on health benefits of vitamin D revisited. Dermato-Endocrinology 4: 183-190.

6 Yanamadala M (2012) Ensuring vitamin D supplementation in nursing home patients--a quality improvement project. J Nutr Gerontol Geriatr 31: 158-171.

7 Costan AR, Vulpoi C, Mocanu V (2014) Vitamin D fortified bread improves pain and physical function domains of quality of life in nursing home residents. J Med Food 17: 625-631.

8 Wimalawansa SJ (2012) Vitamin D in the new millennium. Curr Osteoporos Rep 10: 4-15.

9 Wong TS (2006) A survey of vitamin D level in people with learning disability in long-stay hospital wards in Hong Kong. J Intellect Disabil 10: 47-59.

10 Rolland Y (2013) Vitamin D supplementation in older adults: searching for specific guidelines in nursing homes. J Nutr Health Aging 17: 402-412.

11 Murray CJ (2013) The state of US health, 1990-2010: burden of diseases, injuries, and risk factors. JAMA 310: 591-608.

12 Sullivan WF (2006) Consensus guidelines for primary health care of adults with developmental disabilities. Can Fam Physician 52: 1410-1418.

13 Bischoff FHA (2008) Optimal serum 25-hydroxyvitamin D levels for multiple health outcomes. Adv Exp Med Biol 624: 55-71.

14 Grant WB (2015) Emphasizing the health benefits of vitamin D for those with neurodevelopmental disorders and intellectual disabilities. Nutrients 7: 1538-1564.

15 Kilpinen-Loisa P (2009) Vitamin D status and optimal supplementation in institutionalized adults with intellectual disability. J Intellect Disabil Res 53: 1014-1023.

16 Vanlint S (2008) A guide for the assessment and management of vitamin D status in people with intellectual disability (developed as an AADDM Working Party initiative). J Intellect Dev Disabil 33: 184-188.

17 Perez LFR (2012) EMAS position statement: Vitamin D and postmenopausal health. Maturitas 71: 83-88.

18 Agarwal KS (2002) The impact of atmospheric pollution on vitamin D status of infants and toddlers in Delhi, India. Arch Dis Child 87: 111-113.

19 Wimalawansa SJ (2012) Vitamin D: An essential component for skeletal health. Annals of NYAS 1240: 90-98.

20 Heaney RP (2014) Guidelines for optimizing design and analysis of clinical studies of nutrient effects. Nutr Rev 72: 48-54.

21 Shao T, Klein P (2012) Grossbard, Vitamin D and breast cancer. Oncologist 17: 36-45.
22 Souberbielle JC (2010) Vitamin D and musculoskeletal health, cardiovascular disease, autoimmunity and cancer: Recommendations for clinical practice. Autoimmun Rev 9: 709-715.

23 Ginde AA, Liu MC, Camargo CA (2009) Demographic differences and trends of vitamin D insufficiency in the US population, 1988-2004. Arch Intern Med 169: 626-632.

24 LeBlanc ECR, Fraenkel ZBM, Pappas M (2014) Screening for Vitamin D Deficiency: Systematic Review for the U.S. Preventive Services Task Force Recommendation, A.P.N. 13-05183-EF-1, Editor, Agency for Healthcare Research and Quality: Rockville, MD.

25 Binkley N, Lewiecki EM (2011) Vitamin D and common sense. J Clin Densitom 14: 95-99.

26 Jones G (2008) Pharmacokinetics of vitamin D toxicity. Am J Clin Nutr 88: 582S-586S.

27 Wimalawansa SJ (2012) Vitamin D: Everything You Need to Know. Homagama, Sri Lanka: Karunaratne \& Sons.

28 Huang KE (2014) Surge in US outpatient vitamin D deficiency diagnoses: National Ambulatory Medical Care Survey analysis. South Med J 107: 214-217.

29 Ross AC (2011) The 2011 report on dietary reference intakes for calcium and vitamin D from the Institute of Medicine: what clinicians need to know. J Clin Endocrinol Metab 96: 53-58.

30 American Geriatrics Society Workgroup on Vitamin, D.S.f.O.A., (2014) Recommendations abstracted from the American Geriatrics Society Consensus Statement on vitamin D for Prevention of Falls and Their Consequences. J Am Geriatr Soc 62: 147-152.

31 Pludowski P (2013) Vitamin D effects on musculoskeletal health, immunity, autoimmunity, cardiovascular disease, cancer, fertility, pregnancy, dementia and mortality-a review of recent evidence. Autoimmun Rev 12: 976-989.

32 Taylor CL (2013) Appropriateness of the probability approach with a nutrient status biomarker to assess population inadequacy: a study using vitamin D. Am J Clin Nutr 97: 72-78.

33 Wimalawansa SJ (2012) Vitamin D; What clinicians would like to know. Sri Lanka Journal of Diabetes, Endocrinology and Metabolism 1: 73-88.

34 Luxwolda MF (2013) Vitamin D status indicators in indigenous populations in East Africa. Eur J Nutr 52: 1115-1125.

35 Luxwolda MF (2012) Traditionally living populations in East Africa have a mean serum 25-hydroxyvitamin D concentration of 115 nmol/l. Br J Nutr 108: 1557-1561.

36 Springer NS, Fricke NL (1975) Nutrition and drug therapy for persons with developmental disabilities. Am J Ment Defic 80: 317-322.

37 Prieto $A D$ (2012) Vitamin $D$ threshold to prevent aromatase inhibitorrelated bone loss: the B-ABLE prospective cohort study. Breast Cancer Res Treat 133: 1159-1167.

38 Lappe JM (2007) Vitamin D and calcium supplementation reduces cancer risk: results of a randomized trial. Am J Clin Nutr 85: 1586-1591.

39 Yang L (2013) Therapeutic effect of vitamin D supplementation in a pilot study of Crohn's patients. Clin Transl Gastroenterol 4: e33.

40 Munger KL (2013) Preclinical serum 25-hydroxyvitamin D levels and risk of type 1 diabetes in a cohort of US military personnel. Am J Epidemiol 177: 411-419. 
41 Song Y (2013) Blood 25-hydroxy vitamin D levels and incident type 2 diabetes: a meta-analysis of prospective studies. Diabetes Care 36: $1422-1428$.

42 Quraishi SA (2013) Association between prehospital vitamin D status and hospital-acquired bloodstream infections. Am J Clin Nutr 98 952-959.

43 Pierrot DC, Clerson P, de Paz R, Souberbielle JC (2012) Relationship between $25-\mathrm{OH}-\mathrm{D}$ serum level and relapse rate in multiple sclerosis patients before and after vitamin D supplementation. Ther Adv Neurol Disord 5: 187-198.

44 Petri M (2013) Vitamin D in systemic lupus erythematosus: modest association with disease activity and the urine protein-to-creatinine ratio. Arthritis Rheum 65: 1865-1871.

45 Holick MF (2014) Sunlight, ultraviolet radiation, vitamin D and skin cancer: how much sunlight do we need? Adv Exp Med Biol 810: 1-16.

46 Tangpricha $V$ (2002) Vitamin D insufficiency among free-living healthy young adults. Am J Med 112: 659-662.

47 Holick MF (2002) Vitamin D: the underappreciated D-lightful hormone that is important for skeletal and cellular health. Curr Opin Endo Diabetes 9: 87-98.

48 Holick MF (2007) Optimal vitamin D status for the prevention and treatment of osteoporosis. Drugs Aging, 24(12): 1017-1029.

49 Zabalza R, Múgica I, Sistiaga F, Garrido A, Emparanza JI, et al. (2014) Assessment of vitamin $D$ supplementation in people with intellectual disability. Journal of Intellectual Disability-Diagnosis and Treatment 2: 46-53.

50 Hollis BW (2005) Circulating 25-hydroxyvitamin D levels indicative of vitamin D sufficiency: implications for establishing a new effective dietary intake recommendation for vitamin D. J Nutr 135: 317-322.

51 Annweiler C, Beauchet O (2015) Questioning vitamin D status of elderly fallers and nonfallers: a meta-analysis to address a 'forgotten step'. J Intern Med 277: 16-44.

52 Lips P(2001) Vitamin D deficiency and secondary hyperparathyroidism in the elderly: consequences for bone loss and fractures and therapeutic implications. Endocr Rev 22: 477-501.

53 Barrett CE (2005) Osteoporosis and fracture risk in women of different ethnic groups. J Bone Miner Res 20: 185-194.

54 Valcour A (2012) Effects of age and serum 25-OH-vitamin D on serum parathyroid hormone levels. J Clin Endocrinol Metab 97: 3989-3995.

55 Ginde AA (2012) Defining vitamin D status by secondary hyperparathyroidism in the U.S. population. J Endocrinol Invest 35: 42-48.

56 Bosworth C (2013) Parathyroid hormone and arterial dysfunction in the multi-ethnic study of atherosclerosis. Clin Endocrinol (Oxf) 79: 429-436.

57 Peiris AN, YoussefD, Grant Wb (2012) Secondary hyperparathyroidism: benign bystander or culpable contributor to adverse health outcomes? South Med J 105: 36-42.

58 Naves DM (2011) Calcium, phosphorus, PTH and death rates in a large sample of dialysis patients from Latin America. The CORES Study. Nephrol Dial Transplant 26: 1938-1947.

59 Hagstrom E (2009) Plasma parathyroid hormone and the risk of cardiovascular mortality in the community. Circulation 119: 2765-2771.
60 Schierbeck LL (2011) Parathyroid hormone and vitamin D--markers for cardiovascular and all cause mortality in heart failure. Eur J Heart Fail 13: 626-632.

61 Zhao G (2012) Serum 25-hydroxyvitamin D levels and all-cause and cardiovascular disease mortality among US adults with hypertension: the NHANES linked mortality study. J Hypertens 30: 284-289.

62 Prietl B (2010) Vitamin D supplementation and regulatory T cells in apparently healthy subjects: vitamin D treatment for autoimmune diseases? Isr Med Assoc J 12: 136-139.

63 Zhang HL, Wu J (2010) Role of vitamin D in immune responses and autoimmune diseases, with emphasis on its role in multiple sclerosis. Neurosci Bull 26: 445-454.

64 Veldhuis S (2010) Cancer prevalence in osteoporotic women with low serum vitamin D levels. Menopause 18: 319-322

65 Engel P (2010) Serum 25(OH) vitamin D and risk of breast cancer: a nested case-control study from the French E3N cohort. Cancer Epidemiol Biomarkers Prev 19: 2341-2350.

66 Jenab, M. 2010 Association between pre-diagnostic circulating vitamin D concentration and risk of colorectal cancer in European populations:a nested case-control study. BMJ, 340: b5500.

$67 \mathrm{JiJ}$ (2010) Seasonal and regional variations of asthma and association with osteoporosis: possible role of vitamin D in asthma. J Asthma 47: 1045-1048.

68 DeLuca HF (1990) Osteoporosis and the metabolites of vitamin D. Metabolism 39: 3-9.

69 Pilz S (2009) Vitamin D status and arterial hypertension: a systematic review. Nat Rev Cardiol 6: 621-630.

70 Jasien J (2012) Aging and bone health in individuals with developmental disabilities. Int J Endocrinol 469235.

71 Vanlint S, Nugent M (2006) Vitamin D and fractures in people with intellectual disability. J Intellect Disabil Res 50: 761-767.

72 Dreyfus D, Lauer E, Wilkinson J (2014) Characteristics associated with bone mineral density screening in adults with intellectual disabilities. J Am Board Fam Med 27: 104-114.

73 Morris CA, (1993) Williams Syndrome. In: Pagon RA (Eds.) GeneReviews, Seattle (WA).

74 Bruyere $O$ (2008) Post-fracture management of patients with hip fracture: a perspective. Curr Med Res Opin 24: 2841-2851.

75 Baek JH (2014) Vitamin D levels in children and adolescents with antiepileptic drug treatment. Yonsei Med J 55: 417-421.

76 Meunier $P$ (1996) Prevention of hip fractures by correcting calcium and vitamin D insufficiencies in elderly people. Scand J Rheumatol Suppl 103: 75-80.

77 Carmel AS (2012) The 25(OH)D level needed to maintain a favorable bisphosphonate response is $33 \mathrm{ng} / \mathrm{ml}$. Osteoporos Int 23: 2479-2487.

78 Dhesi JK (2002) Neuromuscular and psychomotor function in elderly subjects who fall and the relationship with vitamin D status. J Bone Miner Res 17: 891-897.

79 Sholas MG, Tann B, Gaebler SD (2005) Oral bisphosphonates to treat disuse osteopenia in children with disabilities: a case series. J Pediatr Orthop 25: 326-331.

80 Lips P (2010) Once-weekly dose of 8400 IU vitamin D(3) compared with placebo: effects on neuromuscular function and tolerability in older adults with vitamin D insufficiency. Am J Clin Nutr 91: 985-991. 
81 Rejnmark L, (2011) Effects of vitamin d on muscle function and performance: a review of evidence from randomized controlled trials. Ther Adv Chronic Dis 2: 25-37.

82 Trivedi DP, Doll R, Khaw KT (2003) Effect of four monthly oral vitamin D3 (cholecalciferol) supplementation on fractures and mortality in men and women living in the community: randomised double blind controlled trial. BMJ 326: 469.

83 Gillespie LD (2009) Interventions for preventing falls in older people living in the community. Cochrane Database Syst Rev 2: CD007146.

84 Staud R (2005) Vitamin D: more than just affecting calcium and bone. Curr Rheumatol Rep 7: 356-364.

85 Bakare MO, Munir KM (2011) Autism spectrum disorders (ASD) in Africa: a perspective. Afr J Psychiatry (Johannesbg) 14: 208-210.

86 Dealberto MJ (2011) Prevalence of autism according to maternal immigrant status and ethnic origin. Acta Psychiatr Scand 123: 339-348.

87 Broe KE (2007) A higher dose of vitamin d reduces the risk of falls in nursing home residents: a randomized, multiple-dose study. J Am Geriatr Soc 55: 234-239.

88 Bischoff FHA (2004) Effect of Vitamin D on falls: a meta-analysis. JAMA 291: 1999-2006.

89 Flicker L (2003) Serum vitamin D and falls in older women in residential care in Australia. J Am Geriatr Soc 51: 1533-1538.

90 Dhesi JK (2002) A rationale for vitamin D prescribing in a falls clinic population. Age Ageing 31: 267-271.

91 Janssen HC, Samson MM, Verhaar HJ (2002) Vitamin D deficiency, muscle function, and falls in elderly people. Am J Clin Nutr 75: 611-615.

92 Bischoff FHA (2004) Vitamin D receptor expression in human muscle tissue decreases with age. J Bone Miner Res 19: 265-269.

93 Pfeifer M, Begerow B, Minne HW (2002) Vitamin D and muscle function. Osteoporos Int 13: 187-194.

94 Bischoff HA (2003) Effects of vitamin D and calcium supplementation on falls: a randomized controlled trial. J Bone Miner Res 18:343-351.

95 Prabhala A, Garg R, Dandona P (2000) Severe myopathy associated with vitamin D deficiency in western New York. Arch Intern Med 160: 1199-1203.

96 Pfeifer M (2000) Effects of a short-term vitamin D and calcium supplementation on body sway and secondary hyperparathyroidism in elderly women. J Bone Miner Res 15: 1113-1118.

97 Graafmans WC (1996) Falls in the elderly: a prospective study of risk factors and risk profiles. Am J Epidemiol 143: 1129-1136.

98 Prince RL (2008) Effects of ergocalciferol added to calcium on the risk of falls in elderly high-risk women. Arch Intern Med 168: 103-108.

99 Meyer HE (2002) Can vitamin D supplementation reduce the risk of fracture in the elderly? A randomized controlled trial. J Bone Miner Res 17: 709-715.

100 Dukas L (2004) Alfacalcidol reduces the number of fallers in a community-dwelling elderly population with a minimum calcium intake of more than $500 \mathrm{mg}$ daily. J Am Geriatr Soc 52: 230-236.

101 Bischoff FHA (2005) Fracture prevention with vitamin D supplementation: a meta-analysis of randomized controlled trials. JAMA 293: 2257-2264.

102 Greenspan SL, Resnick NM, Parker RA (2005) Vitamin D supplementation in older women. J Gerontol A Biol Sci Med Sci 60 754-759.
103 Murad MH (2011) Clinical review: The effect of vitamin D on falls: a systematic review and meta-analysis. J Clin Endocrinol Metab 96: 2997-3006.

104 Bischoff FHA, Orav EJ, Dawson HB (2006) Effect of cholecalciferol plus calcium on falling in ambulatory older men and women: a 3-year randomized controlled trial. Arch Intern Med 166: 424-430.

105 Richy F, Dukas L, Schacht E (2008) Differential effects of D-hormone analogs and native vitamin $D$ on the risk of falls: a comparative meta-analysis. Calcif Tissue Int 82: 102-107.

106 Schaller F (2012) Mild to moderate cognitive impairment is a major risk factor for mortality and nursing home admission in the first year after hip fracture. Bone 51: 347-352.

107 Heaney R (2015) Astatistical error in the estimation of the recommended dietary allowance for vitamin D. Nutrients 7: 1688-1690.

108 Veugelers PJ, Ekwaru JP (2014) A statistical error in the estimation of the recommended dietary allowance for vitamin D. Nutrients 6: $4472-4475$

109 Grant WB (2011) An estimate of the global reduction in mortality rates through doubling vitamin D levels. Eur J Clin Nutr 65: 1016-1026.

110 Grant WB (2012) Ecological studies of the UVB-vitamin D-cancer hypothesis. Anticancer Res 32: 223-236.

111 Binkley N (2009) Is vitamin D the fountain of youth? Endocr Pract 15: 590-596.

112 Norman AW (2007) 13th Workshop consensus for vitamin D nutritional guidelines. J Steroid Biochem Mol Biol 103: 204-205.

113 Binkley N, (2007) Does low vitamin D status contribute to "agerelated" morbidity? J Bone Miner Res 22 : 55-58.

114 Holick MF (2006) High prevalence of vitamin D inadequacy and implications for health. Mayo Clin Proc 81: 353-373.

115 Holick MF (2008) Vitamin D: a D-Lightful health perspective. Nutr Rev 66: 182-194.

116 Reddy VS (2010) Role of vitamin D in cardiovascular health. Am J Cardiol 106: 798-805.

117 Chung M (2009) Vitamin D and calcium: a systematic review of health outcomes. Evid Rep Technol Assess 183: 1-420.

118 Manson JE, Mayne ST, Clinton SK (2011) Vitamin D and prevention of cancer--ready for prime time? N Engl J Med 364: 1385-1387.

119 Autier P (2014) Vitamin D status and ill health: a systematic review. Lancet Diabetes Endocrinol 2: 76-89.

120 Bolland MJ (2014) The effect of vitamin D supplementation on skeletal, vascular, or cancer outcomes: a trial sequential metaanalysis. Lancet Diabetes Endocrinol 2: 307-320.

121 Bischoff HA (2001) In situ detection of 1,25-dihydroxyvitamin D3 receptor in human skeletal muscle tissue. Histochem J 33: 19-24.

122 Edvardsen K (2011) Vitamin D-effective solar UV radiation, dietary vitamin D and breast cancer risk. Int J Cancer 128: 1425-1433.

123 Engel P (2011) Joint effects of dietary vitamin D and sun exposure on breast cancer risk: Results from the French E3N Cohort. Cancer Epidemiol Biomarkers Prev 20: 187-198.

124 Grant WB (2011) Vitamin D levels in Norway may be inadequate to reduce risk of breast cancer. Int J Cancer 128: 2249-2250.

125 Grant WB, Garland CF (2002) Evidence supporting the role of vitamin $D$ in reducing the risk of cancer. J Intern Med 252: 178-180. 
126 Grant WB (2002) An estimate of premature cancer mortality in the U.S. due to inadequate doses of solar ultraviolet-B radiation. Cancer 94: $1867-1875$

127 Garland CF (2006) The role of vitamin D in cancer prevention. Am J Public Health 96: 252-261.

128 Baron JA (1999) Calcium supplements for the prevention of colorectal adenomas. Calcium Polyp Prevention Study Group. N Engl J Med 340: 101-107.

129 Deeb KK, Trump DL, Johnson CS (2007) Vitamin D signalling pathways in cancer: potential for anticancer therapeutics. Nat Rev Cancer 7: 684-700.

130 Giovannucci E (2006) Prospective study of predictors of vitamin D status and cancer incidence and mortality in men. J Natl Cancer Inst 98: 451-459.

131 Liu PT (2006) Toll-like receptor triggering of a vitamin D-mediated human antimicrobial response. Science 311: 1770-1773.

132 Liu N (2009) Vitamin D induces innate antibacterial responses in human trophoblasts via an intracrine pathway. Biol Reprod 80: 398-406.

133 Grant WB (2011) A review of the role of solar ultraviolet-B irradiance and vitamin $D$ in reducing risk of dental caries. Dermatoendocrinol 3: 193-198.

134 Mascitelli L, Pezzetta F, Goldstein MR (2011) Inflammatory bowel disease and the vitamin D endocrine system. Intern Med J 41: 369-370.

135 Raman M (2011) Vitamin D and gastrointestinal diseases: inflammatory bowel disease and colorectal cancer. Therap Adv Gastroenterol 4: 49-62.

136 Ardizzone S (2011) Vitamin D and inflammatory bowel disease. Vitam Horm 86: 367-377.

137 Bentley RW (2011) Vitamin D receptor gene polymorphism associated with inflammatory bowel disease in New Zealand males. Aliment Pharmacol Ther 33: 855-856.

138 Smith PA (2006) Nutrition, hydration, and dysphagia in long-term care: Differing opinions on the effects of aspiration. J Am Med Dir Assoc 7: 545-549.

139 Corcoran L, (2005) Nutrition and hydration tips for stroke patients with dysphagia. Nurs Times 101: 24-27.

140 Wimalawansa SJ (2016) Vitamin D deficiency Is a surrogate marker for visceral fat content, metabolic syndrome, type 2 diabetes, and future metabolic complications. J Diabetes, Metabolic Disorders \& Control 3: 1-10.

141 Afzal S (2014) Plasma 25-hydroxyvitamin D, lung function and risk of chronic obstructive pulmonary disease. Thorax 69: 24-31.

142 Sundar IK, Rahman I (2011) Vitamin d and susceptibility of chronic lung diseases: role of epigenetics. Front Pharmacol 2: 50.

143 Gezen AD, Dursun E, Yilmazer S (2013) Vitamin D inquiry in hippocampal neurons: consequences of vitamin D-VDR pathway disruption on calcium channel and the vitamin $D$ requirement. Neurol Sci 34: 1453-1458.

144 Gezen AD, Yilmazer S, Dursun E (2014) Why vitamin D in Alzheimer's disease? The hypothesis. J Alzheimers Dis 40: 257-269.

145 Cannell JJ, Grant WB (2013) What is the role of vitamin D in autism? Dermatoendocrinol 5: 199-204.

146 Cannell JJ (2008) Autism and vitamin D. Med Hypotheses 70: 750-759.
147 Patrick RP, Ames BN (2014) Vitamin D hormone regulates serotonin synthesis. Part 1: relevance for autism. FASEB J 28: 2398-2413.

148 Neece CL,Blacher BBJ, Crnic KA(2011) Attention-deficit/hyperactivity disorder among children with and without intellectual disability: an examination across time. J Intellect Disabil Res 55: 623-635.

149 Bener A, Kamal M (2014) Predict attention deficit hyperactivity disorder? Evidence -based medicine. Glob J Health Sci 6: 47-57.

150 Goksugur SB (2014) Vitamin D status in children with attentiondeficit-hyperactivity disorder. Pediatr Int 56: 515-519.

151 Rucklidge JJ (2014) Moderators of treatment response in adults with ADHD treated with a vitamin-mineral supplement. Prog Neuropsychopharmacol Biol Psychiatry 50: 163-171.

152 Hujoel PP (2013) Vitamin D and dental caries in controlled clinical trials: systematic review and meta-analysis. Nutr Rev 71: 88-97.

153 Mellanby M, Pattison CL (1928) The Action of Vitamin D in Preventing the Spread and Promoting the Arrest of Caries in Children. Br Med J 2: 1079-1082.

154 Krall EA (2001) Calcium and vitamin D supplements reduce tooth loss in the elderly. Am J Med, 111(6): 452-6.

155 Dietrich T (2004) Association between serum concentrations of 25-hydroxyvitamin D3 and periodontal disease in the US population. Am J Clin Nutr 80: 108-113.

156 Pierrot DC (2009) Clinical implications of a possible role of vitamin D in multiple sclerosis. J Neurol 256: 1468-1479.

157 Mikosch P (2009) Patients with Gaucher disease living in England show a high prevalence of vitamin D insufficiency with correlation to osteodensitometry. Mol Genet Metab 96: 113-1120.

158 Zittermann A (2012) Vitamin D deficiency and mortality risk in the general population: a meta-analysis of prospective cohort studies. Am J Clin Nutr 95: 91-100.

159 Dror Y (2013) Vitamin D levels for preventing acute coronary syndrome and mortality: evidence of a nonlinear association. J Clin Endocrinol Metab 98: 2160-2167.

160 Ensrud KE (2011) Circulating 25-hydroxyvitamin D levels and frailty in older men: the osteoporotic fractures in men study. J Am Geriatr Soc 59: 101-106.

161 Ensrud KE (2010) Circulating 25-hydroxyvitamin D levels and frailty status in older women. J Clin Endocrinol Metab 95: 5266-5273.

162 Grant WB (2010) Relation between prediagnostic serum 25-hydroxyvitamin D level and incidence of breast, colorectal, and other cancers. J Photochem Photobiol B 101: 130-136.

163 Turner AG, Anderson PH, Morris HA 2012 Vitamin D and bone health. Scand J Clin Lab Invest Suppl 243: 65-72.

164 Spedding S (2013) Does vitamin D sufficiency equate to a single serum 25-hydroxyvitamin d level or are different levels required for non-skeletal diseases? Nutrients 5: 5127-5139.

165 Ford ES (2011) Vitamin D and all-cause mortality among adults in USA: findings from the National Health and Nutrition Examination Survey Linked Mortality Study. Int J Epidemiol 40: 998-1005.

166 Schottker B (2013) Strong associations of 25-hydroxyvitamin D concentrations with all-cause, cardiovascular, cancer, and respiratory disease mortality in a large cohort study. Am J Clin Nutr 97: 782-793. 
167 Bischoff FHA (2009) Fall prevention with supplemental and active forms of vitamin D: a meta-analysis of randomised controlled trials. BMJ 339: b3692.

168 Gillespie LD (2012) Interventions for preventing falls in older people living in the community. Cochrane Database Syst Rev 9: CD007146.

169 Sokol SI (2011) Vitamin D status and risk of cardiovascular events: lessons learned via systematic review and meta-analysis. Cardiol Rev 19: 192-201.

170 Ginde AA (2009) Prospective study of serum 25-hydroxyvitamin D level, cardiovascular disease mortality, and all-cause mortality in older U.S. adults. J Am Geriatr Soc 57: 1595-1603.

171 Garland CF (2009) Vitamin D for cancer prevention: global perspective. Ann Epidemiol 19: 468-483.

172 Urashima M (2010) Randomized trial of vitamin D supplementation to prevent seasonal influenza A in schoolchildren. Am J Clin Nutr 91: $1255-1260$

173 Martineau AR (2011) High-dose vitamin D(3) during intensive-phase antimicrobial treatment of pulmonary tuberculosis: a double-blind randomised controlled trial. Lancet 377: 242-250.

174 Forouhi NG (2012) Circulating 25-hydroxyvitamin D concentration and the risk of type 2 diabetes: results from the European Prospective Investigation into Cancer (EPIC)-Norfolk cohort and updated metaanalysis of prospective studies. Diabetologia 55: 2173-2182.

175 Jorde R (2008) Effects of vitamin D supplementation on symptoms of depression in overweight and obese subjects: randomized double blind trial. J Intern Med 264: 599-609.

176 Hansen KE (2011) High-dose vitamin D: helpful or harmful? Curr Rheumatol Rep 13: 257-264.
177 Deluca HF, Prahl JM, Plum LA, 2011 1,25-Dihydroxyvitamin D is not responsible for toxicity caused by vitamin D or 25-hydroxyvitamin D. Arch Biochem Biophys 505: 226-230.

178 Sanders KM (2010) Annual high-dose oral vitamin D and falls and fractures in older women: a randomized controlled trial. JAMA 303: 1815-1822.

179 Lyons RA (2007) Preventing fractures among older people living in institutional care: a pragmatic randomised double blind placebo controlled trial of vitamin D supplementation. Osteoporos Int 18 : 811-818.

180 Law M (2006) Vitamin D supplementation and the prevention of fractures and falls: results of a randomised trial in elderly people in residential accommodation. Age Ageing 35: 482-486.

181 Wimalawansa, SJ (2016) Optimum duration and safety of long-term use of potent anti-resorptive medications in osteoporosis. Expert Rev Endocrinol Metab E-Pub.

182 Wimalawansa SJ (2013) Food fortification programs to alleviate micronutrient deficiencies. J. Food Process Technol 4: 257-267.

183 LeBlanc E (2014) in Screening for Vitamin D Deficiency: Systematic Review for the U.S. Preventive Services Task Force Recommendation, Rockville (MD).

184 Rao DS (2002) Role of vitamin D and calcium nutrition in disease expression and parathyroid tumor growth in primary hyperparathyroidism: a global perspective. J Bone Miner Res 17: 75-80.

185 Rao DS (2000) Ef fect of vitamin D nutrition on parathyroid adenoma weight: pathogenetic and clinical implications. J Clin Endocrinol Metab 85: 1054-1058.

186 Holick MF (2007) Vitamin D deficiency. N Engl J Med 357: 266-281. 